\title{
FÓRMULAS INFANTIS INDUSTRIALIZADAS: impacto na informação nutricional
}

\author{
Evellyn de Oliveira PANIAGO \\ evellyn.paniago@hotmail.com \\ Ulana Chaves SARMENTO ${ }^{\mathrm{b}}$ \\ ulanachaves@hotmail.com \\ Osmar Ferreira de ANDRADE ${ }^{\mathrm{b}}$ \\ andradedta@gmail.com \\ July Elen Vilas BOAS ${ }^{a}$ \\ july_ellem@hotmail.com \\ Priscila Silva FIGUEIREDO ${ }^{c}$ \\ pri.figueiredo92@gmail.com
}

Rita de Cássia Avellane da GUIMARÃES

rita.guimaraes@ufms.br*(autora para correspondência)

\begin{abstract}
aAcadêmica do curso de Nutrição da Universidade Federal de Mato Grosso do Sul, UFMS, 79900-000, Campo Grande, MS, Brazil.

${ }^{\text {b}}$ Técnica da Unidade de Tecnologia de Alimentos e Saúde Pública (UTASP) da Universidade Federal de Mato Grosso do Sul, UFMS, Faculdade de Medicina (FAMED), Pós Graduação em Saúde e Desenvolvimento na Região CentroOeste, 79900-000, Campo Grande, MS, Brasil.

c Mestranda da Universidade Federal de Mato Grosso do Sul, UFMS, Faculdade de Medicina (FAMED), Pós Graduação em Saúde e Desenvolvimento na Região Centro-Oeste, 79900-000, Campo Grande, MS, Brasil.

${ }^{\text {d }}$ Docente da Universidade Federal de Mato Grosso do Sul, UFMS, Faculdade de Medicina (FAMED), Pós Graduação em Saúde e Desenvolvimento na Região Centro-Oeste, 79900-000, Campo Grande, MS, Brasil.
\end{abstract}

Recebido em: 14/05/2016 - Aprovado em: 14/11/2017 - Disponibilizado em: 30/12/2017

\begin{abstract}
RESUMO
A adequação nutricional da fórmula infantil deve ser cientificamente comprovada a fim de garantir o crescimento e desenvolvimento da criança. O objetivo do presente estudo foi analisar a composição de fórmulas infantis, comparandoas com a informação nutricional disponível no rótulo do produto, com finalidade de verificar adequação da composição química. No presente trabalho avaliou-se sete fórmulas infantis para faixa etária de 6 a 10 meses de idade, obtidas no comércio de Campo Grande-MS. Para o efeito, analisou-se o teor de umidade, cinzas, carboidratos, proteínas, lipídeos e valor energético total, através dos métodos oficiais de análise. Quanto ao teor energético, a formulação F6 foi a que mais diferiu-se da informação do rótulo, enquanto que F3 foi a que mais assemelhou-se do rótulo. Quanto aos valores de proteínas e lipídeos obtiveram valores próximos aos fornecidos pelo rótulo. Já os carboidratos apresentaram

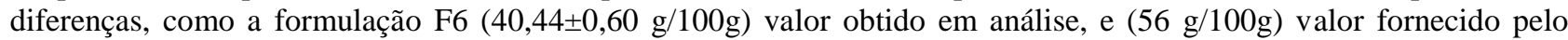
rótulo, a mesma apresentou o menor valor dentre as formulações. Evidencia-se que as fórmulas infantis são substitutas ao leite materno e são nutricionalmente seguras, no entanto apontam a necessidade de um contínuo monitoramento desses produtos, uma vez que a inadequação dos nutrientes do teor lipídico e outros pode afetar de maneira significativa o desenvolvimento infantil.
\end{abstract}

Palavras-chave: Criança.Adequação nutricional.Composição centesimal. Rótulo. Segurança alimentar.

\begin{abstract}
The nutritional adequacy of infant formula shall be scientifically proven to ensure the growth and development of children. The aim of this study was to analyze the composition of infant formula, comparing them with nutrition information available on the product label, with purpose of verifying adequacy of the chemical composition. In the present study we evaluated seven infant formula for age 6-10 months old, obtained in trade in Campo Grande-MS. To this end, we analyzed the moisture content, ash, carbohydrates, proteins, lipids and total energy with the official methods of analysis. As for the energy content formulation F6 was the most differed from the label information, while F3 was the most resembled the label. As for the values of proteins and lipids obtained values close to those provided by
\end{abstract}


the label. Have carbohydrates significantly different, as the F6 formulation $(40.44 \pm 0.60 \mathrm{~g} / 100 \mathrm{~g})$ value obtained from analysis, and (56 g / $100 \mathrm{~g}$ ) value provided by the label, it presented the lowest value among the formulations. Evidence that infant formulas are substitute to breast milk and are nutritionally safe, however highlight the need for continuous monitoring of these products, since the inadequacy of nutrients and other lipid content can significantly affect child development.

Keywords: Child.Infantformulas.Nutritionaladequacy.Chemicalcomposition.Label.Foodsafety.

\section{Introdução}

A infância é um período de extrema importância para o crescimento e desenvolvimento. Segundo o Estatuto da Criança e do Adolescente (ECA) é considerada criança, aquela com doze anos incompletos. $\mathrm{O}$ artigo $4^{\circ}$ doECA prevê como dever da família, da comunidade, da sociedade em geral e do poder público assegurar, com absoluta prioridade, a efetivação dos direitos referentes à vida, à saúde, à alimentação, à educação, ao esporte, ao lazer, à profissionalização, à cultura, à dignidade, ao respeito, à liberdade e à convivência familiar e comunitária(BRASIL,1990).

A alimentação correta no primeiro ano de vida tem grande importância para $\mathbf{o}$ desenvolvimento da criança; dela vai depender em grande parte o seu futuro. A alimentação infantil adequada compreende a prática do aleitamento materno e a introdução de alimentos apropriados, em tempo oportuno, que complementam o aleitamento (BRASIL, 2002).

$\mathrm{O}$ leite materno possui todos os nutrientes necessários para o crescimento e desenvolvimento ótimos do bebê, suprindo nos primeiros seis meses de vida e atuando de maneira importante como fonte de nutrientes, especialmente de proteínas, gorduras e vitaminas, no segundo ano de vida (BRASIL, 2009).No entanto em alguns casos o aleitamento materno não é possível, e então as fórmulas infantis vêm como substitutivas do leite materno, sendo a rotulagem nutricional de grande importância.A importância da rotulagem nutricional dos alimentos para a promoção da alimentação saudável é destacada em grande parte dos estudos e pesquisas que envolvem a área da nutrição e sua relação com estratégias para a redução do risco de doenças crônicas(BRASIL, 1999).Na rotulagem nutricional é obrigatório constar informações quantitativas a respeito do valor energético, carboidratos, proteínas, gorduras totais, gorduras saturadas, gorduras trans, fibra alimentar e sódio (ANVISA, 2005).

A Resolução da Agência Nacional de Vigilância Sanitária (Anvisa), RDC no 360de 23 de dezembro de2003, trata do regulamento técnico a respeito da rotulagem nutricional de alimentos embalados, torna obrigatória a 
rotulagem nutricional baseada nas regras estabelecidas com o objetivoprincipal de atuar em benefício do consumidor e ainda evitar obstáculos técnicos ao comércio (BRASIL, 2003).

A fórmula infantil é um produto à base de leite de vaca, ou de leite de outros animais,e/ou de outros ingredientes que foram aprovados para serem adequados à alimentação infantil. A segurança nutricional e a adequação da fórmula infantil devem ser cientificamente comprovadas, a fim de garantir o crescimento e desenvolvimento normais da criança (PALMER, 2009).

Com intuito de avaliar a composição nutricional de fórmulas infantis comercializadas justifica-se no presente estudo, analisara composição de fórmulasinfantis de lactentes de 6 meses a 10 meses, comparando-as com a informação nutricional disponível no produto com finalidade de verificar adequação do valor calórico e de macronutrientes existentes nestas dietas pediátricas.

\section{MATERIAL E MÉTODOS}

\section{Obtenção das fórmulas infantis}

Fórmulasinfantis recomendadas para lactentes de 6 a 10 meses foram obtidas no comércio da cidade de Campo Grande, MS, totalizando 7 dietas.

\section{Composição centesimal}

As análises das fórmulas infantis foram realizadas no laboratório de físicoquímica da Unidade de Tecnologia de Alimentos e Saúde Pública (UTASP) da Universidade Federal de Mato Grosso do Sul (UFMS).

A composição química das amostras coletadasfoi realizada em triplicata, de acordo com as normas analíticas do Instituto Adolfo Lutz (INSTITUTO ADOLFO LUTZ, 2005). A análise da umidade foi realizada em estufa a $105{ }^{\circ} \mathrm{C}$ até peso constante. A análise de resíduo mineral fixo foi realizada por incineração em mufla a $550{ }^{\circ} \mathrm{C}$. O teor de lipídios foi determinado utilizando-se Lactobutirômetro de Gerber (AOAC, 2010). O teor de proteínasfoi determinado pelo conteúdo de nitrogênio total, segundo método do micro Kjedahl e o fator de 6,38 usado para a conversão do teor de nitrogênio em proteína bruta.

Para a análise de carboidratos foi realizada a determinação da porcentagem de glicídios redutores em lactose (INSTITUTO ADOLFO LUTZ, 2005).O valor energético total proveniente dos nutrientes foi expresso em quilocalorias (kcal), estimada a partir dos fatores de conversão de Atwater: $\mathrm{kcal}=(4 \mathrm{x} \mathrm{g}$ proteína $)+(4 \times \mathrm{g}$ carboidratos $)+(9 \mathrm{x} \mathrm{g}$ lipídios) (MERRIL; WATT, 1973). As análises foram realizadas no mês de julho de 2014. 


\section{Análise estatística}

Os dados foramanalisados através do software Statistical Package for the Social Sciences (SPSS), versão 22.0, através da análise de variância (ANOVA) pelo teste de Tukey. Os valores foram considerados significantes quando $p<0,05$.

\section{RESULTADOS E DISCUSSÃO}

Os resultados da composição estão reportados na Tabela 1 e Tabela 2 . A fórmula infantil de seguimento para lactentes é o produto em forma líquida ou em pó utilizado, quando indicado, como substituto do leite materno ou humano a partir do sexto mês. Normalmente é um produto à base de leite de vaca, ou de leite de outros animais. 
Tabela 1 - Composição química $(\mathrm{g} / 100 \mathrm{~g})$ e calorias $(\mathrm{kcal} / 100 \mathrm{~g})$ de Fórmulas infantis para crianças de 6 a 10 meses, coletados em diferentes estabelecimentos da cidade de Campo Grande, MS.

\begin{tabular}{|c|c|c|c|c|c|c|c|c|c|c|}
\hline FORMULAÇÃO & UMIDADE & CINZAS & PROTEÍNAS & REF*. & LIPÍDEOS & REF*. & CARBOIDRATOS & REF*. & CALORIAS & REF*. \\
\hline $\mathrm{F} 1$ & $2,44 \pm 0,15^{\mathrm{a}}$ & $3,49 \pm 0,00^{\mathrm{a}}$ & $16,72 \pm 0,14^{\mathrm{ab}}$ & 15 & $22,06 \pm 0,03^{c}$ & 22 & $46,14 \pm 3,02^{\mathrm{bc}}$ & 58 & $444,03 \pm 6,53^{b}$ & 483 \\
\hline $\mathrm{F} 2$ & $1,39 \pm 0,06^{\mathrm{b}}$ & $3,19 \pm 0,00^{\mathrm{c}}$ & $16,85 \pm 0,44^{\mathrm{a}}$ & 15 & $22,07 \pm 1,03^{\mathrm{c}}$ & 21 & $59,37 \pm 5,03^{\mathrm{a}}$ & 58 & $494,42 \pm 25,98^{\mathrm{ab}}$ & 482 \\
\hline F3 & $1,60 \pm 0,02^{\mathrm{b}}$ & $3,18 \pm 0,02^{\mathrm{c}}$ & $15,50 \pm 1,90^{\mathrm{abc}}$ & 15 & $21,80 \pm 0,47^{\mathrm{c}}$ & 21 & $53,84 \pm 0,51^{\mathrm{ab}}$ & 54 & $471,62 \pm 11,17^{\mathrm{ab}}$ & 469 \\
\hline $\mathrm{F} 4+$ & $2,31 \pm 0,02^{\mathrm{a}}$ & $2,79 \pm 0,00^{\mathrm{d}}$ & $13,52 \pm 0,32^{\mathrm{cd}}$ & 12 & $24,10 \pm 0,83^{\mathrm{bc}}$ & 23 & $57,61 \pm 1,21^{\mathrm{ab}}$ & 59 & $511,26 \pm 4,28^{\mathrm{a}}$ & 493 \\
\hline F5 & $1,57 \pm 0,12^{\mathrm{b}}$ & $3,15 \pm 0,00^{\mathrm{c}}$ & $14,51 \pm 0,76^{\mathrm{bcd}}$ & 14 & $22,61 \pm 0,91^{\mathrm{c}}$ & 21 & $59,79 \pm 5,73^{\mathrm{a}}$ & 53 & $490,14 \pm 3,70^{\mathrm{ab}}$ & 457 \\
\hline F6 & $2,47 \pm 0,05^{\mathrm{a}}$ & $2,14 \pm 0,02^{\mathrm{e}}$ & $13,56 \pm 0,43^{\mathrm{cd}}$ & 13 & $25,69 \pm 0,11^{\mathrm{ab}}$ & 26 & $40,44 \pm 0,60^{\mathrm{c}}$ & 56 & $446,35 \pm 0,88^{b}$ & 506 \\
\hline F7 & $1,58 \pm 0,03^{\mathrm{b}}$ & $3,35 \pm 0,02^{\mathrm{b}}$ & $12,95 \pm 0,13^{\mathrm{d}}$ & 12 & $28,08 \pm 0,22^{\mathrm{a}}$ & 28 & $56,67 \pm 4,81^{\mathrm{ab}}$ & 55 & $533,69 \pm 29,35^{\mathrm{a}}$ & 519 \\
\hline
\end{tabular}

REF*:Rótulo das fórmulas analisadas. **Letras diferentes na mesma coluna indicam diferença estatística pelo teste de Tukey $(p<0,05)$. + CHO calculado por diferença $100-$ (Umidade+PTN+LIP+CHO). 
Tabela 2 - Composição química $(\mathrm{g} / 100 \mathrm{~g})$ e calorias $(\mathrm{kcal} / 100 \mathrm{~g})$ por $100 \mathrm{ml}$ de Fórmulas infantis reconstituídas para crianças de 6 a 10 meses, coletados em diferentes estabelecimentos da cidade de Campo Grande, MS

\begin{tabular}{|c|c|c|c|c|c|c|c|c|}
\hline FORMULAÇÃO & PROTEÍNAS & REF*. & LIPÍDEOS & REF*. & CARBOIDRATOS & REF*. & CALORIAS & REF*. \\
\hline $\mathrm{F} 1$ & 2,32 & 2,0 & 3,06 & 3,0 & 6,41 & 8,0 & 61,72 & 67,0 \\
\hline $\mathrm{F} 2$ & 2,34 & 2,1 & 3,06 & 2,9 & 8,25 & 8,1 & 68,72 & 67,0 \\
\hline F3 & 2,21 & 2,1 & 3,11 & 3,1 & 7,69 & 7,7 & 67,44 & 67,0 \\
\hline $\mathrm{F} 4$ & 1,86 & 1,7 & 3,32 & 3,2 & 7,95 & 8,1 & 70,55 & 68,0 \\
\hline F5 & 2,14 & 2,0 & 3,34 & 3,1 & 8,84 & 7,9 & 75,54 & 68,0 \\
\hline F6 & 1,78 & 1,7 & 3,39 & 3,4 & 5,33 & 7,3 & 58,91 & 67,0 \\
\hline F7 & 1,70 & 1,5 & 3,69 & 3,7 & 7,46 & 7,2 & 70,28 & 68,0 \\
\hline
\end{tabular}

REF*:Rótulo das fórmulas analisadas 
Quanto ao teor energético as formulações, F2, F3 e F4, não se diferiram entre si, e nem diferiram-se das demais formulações. A formulação $\mathrm{F} 1$ e $\mathrm{F} 6$ apresentou teor energético mais baixo em relação às demais $(444,06$ e $446,35 \mathrm{kcal} / 100 \mathrm{~g}$, respectivamente), porém não diferiram estatisticamente entre si. Ao contrário da formulação F4 e F7 que apresentou valor energético superior quando comparada as demais. Observa-se ainda que apesar das fórmulas serem para um mesmo público alvo, e apresentarem mesma finalidade, algumas delas acabam oferecendo maior aporte calórico em relação a outras. Através dos valores de referência obtidos nos rótulos das fórmulas, nota-se diferença entre os resultados obtidos em análise e os fornecidos pelo rótulo como, por exemplo, a formulação F3 que apresentou valor próximo ao de referência, no entanto, nas demais verificou-se valores discrepantes.

Resultados encontrados em F1, F2, F3 e F6 estão dentro da recomendação preconizado pela Resolução da Agência Nacional de Vigilância Sanitária (Anvisa), RDC nº44de 19 de setembro de2011, para valor energético referido para este produto, reconstituído de, no mínimo 60 kcal (250 kJ) e no máximo 70 kcal (295kJ). Dentre a formulações verifica-se que a F4, F5 e
F7(70,55, 75,54 e 70,28 kcal) ultrapassa o valor de referência (BRASIL, 2011).

Quanto ao baixo teor de umidade, justifica-se pelo fato de ser um produto em pó (sem adição de água). Observa-se que as formulações F1, F4, e F6 diferiram-se estatisticamente das demais, e apresentaram maior teor de umidade. Entretanto, F2, F3 e F5 apresentaram menor teor de umidade $(1,39 \%, 1,60 \%$, 1,57\%, respectivamente). Tavares (2013)analisou a composição química de fórmulas infantis e verificou teor de umidade de 2,63\% $\pm 0,47$ variando até $3,54 \% \pm 0,49$, o que assemelha-se do presente estudo.

Erhinyodavweet al. determinaram o teor de umidade em diferentes fórmulas lácteas, e constataramvalores entre 0,90 a $2,45 \%$. Semeniuc e colaboradores (2012)referem valores entre $2,25 \%$ e $3,75 \%$ para fórmulas lácteas, e estipulam ainda um limite máximo no teor de umidade na ordem de3\%. Fitzpatricket al.(2005) analisaram dois tipos de leites em pó, e referem valores na ordem de $3,3 \%$ e $4,7 \%$.

Para as cinzas, na formulação F1 obteve-se resultado de $3,49 \mathrm{~g} / 100 \mathrm{~g}$, enquanto que, F6 quando comparada as demais, apresentou menor valor $(2,14$ $\mathrm{g} / 100 \mathrm{~g}$ ). As cinzas são referidas como o 
teor de minerais presentes em um alimento, podendo, a partir de analisar-se sódio, potássio, cálcio, ferro, magnésio, manganês, fósforo entre outros. Segundo Ekpeteet al. (2013), o teor de cinza em fórmulas lácteas está compreendidoentre $2,30 \%$ a 3,56\%. Assim valores obtidos nesta análise estão de acordo com os valores reportados pelos autores.

A formulação F7 apresentou menor valor para proteínas, quando reconstituída $(1,70 \mathrm{~g} / 100 \mathrm{~g})$, diferindo-se da formulação F2que apresentou o maior teor de proteínas $(16,85 \mathrm{~g} / 100 \mathrm{~g})$, quando reconstituída $(2,34$ g/100 g). Ao analisar com os valores de referência preconizados nos rótulos das fórmulas, não verificou-se diferenças discrepantes entre os valores obtidos em análise.

Tavares

(2013)

realizou composição química de quatro fórmulas infantis, onde apresentaram teor de proteína variando de 2,38g/100 g a 3,11 g/100 g, sendo possível observar que os valores de proteínas das formulações analisadas estão abaixo, no entanto próximos. Verificado na recomendação da Resolução da Anvisa, RDC n44de 19 de setembro de2011, referida para este produto reconstituído quanto ao teor de proteína, reporta-se como valor mínimo 1,8 g/100g, e máximo de 3,5 g/100g, assim as fórmulas analisadas seguem a recomendação, com exceção das formulações F6 e F7, que apresentaram teores de proteínas abaixo do estabelecido $(1,78$ e $1,70 \mathrm{~g} / 100 \mathrm{~g}$, respectivamente $)$ (BRASIL, 2011).

Em relação ao teor de lipídeos, a formulação F7 apresentou-se superior $(28,08 \mathrm{~g} / 100 \mathrm{~g})$ quando reconstituído $(3,69$ $\mathrm{g} / 100 \mathrm{~g}$ ), enquanto que, F1, F2, F3 e F5 apresentaram os menores valores $(22,06$; $22,07 ; 21,80 ; 22,61 \mathrm{~g} / 100 \mathrm{~g})$ quando reconstituído $(3,06 ; 3,06 ; 3,11 \mathrm{~g} / 100 \mathrm{~g})$, não diferindo-se estatisticamente entre si. No estudo realizado por Tavares (2013)com quatro fórmulas infantis, observa-se variação de 4,21 g/100 g a 5,20 $\mathrm{g} / 100 \mathrm{~g}$, verificando-se que os valores encontrados para lipídeos, no presente trabalho, foram pouco acima do reportado. Já quando confrontado com os valores fornecidos pelo rótulo percebe-se que todas formulações analisadas condizem com o que o rótulo refere.

A RDC no44de 19 de setembro de2011 da Anvisa refere para este produto reconstituído, que o mínimo de gorduras totais deve estar entre $4,0 \mathrm{~g} / 100 \mathrm{~g}$ a 6,0 g/100g (BRASIL, 2011). No presente estudo, verificou-se que todas as formulações apresentaram-se abaixo do estipulado pela resolução. 
Em relação ao teor de carboidratos, verifica-se que F2 e F5 apresentaram teores mais elevados de carboidratos $(59,37$ e $59,79 \mathrm{~g} / 100 \mathrm{~g})$, quando reconstituído $(8,25$ e $8,84 \mathrm{~g} / 100 \mathrm{~g})$ respectivamente; já a formulação F6 apresentou menor teor $(40,44 \mathrm{~g} / 100 \mathrm{~g})$, quando reconstituído $(5,33 \mathrm{~g} / 100 \mathrm{~g})$. Quando analisados com os valores obtidos nos rótulos das fórmulas, verifica-se uma diferença de algumas formulações entre os valores aqui obtidos, a formulação F6 apresentando a maior diferença $(40,44 \pm 0,60 \mathrm{~g} / 100 \mathrm{~g})$ valor obtido em análise e $(56 \mathrm{~g} / 100 \mathrm{~g})$ valor fornecido pelo rótulo. Além disso, quando compara-se com a recomendação estabelecida pela Anvisa, (RDC no44de 19 de setembro de2011), referida para este produto reconstituído, o teor mínimo de carboidratos totais deve ser de 9,0 g/100g, e o máximo de $14,0 \mathrm{~g} / 100 \mathrm{~g}$, sendo que o teor mínimo de lactose deve ser de 4,5 g por $100 \mathrm{~g}$ do produto pronto para consumo. Assim as amostras analisadas no presente estudo estão de acordo com o estabelecido pela resolução(BRASIL, 2011).

Quando se compara os resultados obtidos das análises das fórmulas infantis, com outros leites também são usados pela mesma faixa etária na Tabela de Composição Nutricional dos Alimentos
Consumidos no Brasil (IBGE, 2011), como o leite de vaca integral, encontra-se valores próximos do valor energético $(60,03$ kcal/100g); para carboidratos, os F2, F4 e F5 os valores obtidos na análise foram dobro do fornecido pela tabela em questão $(4,52 \mathrm{~g} / 100 \mathrm{~g})$. Em relação ao teor de proteínas, os valores estão próximos aos fornecidos pela tabela $(3,22 \mathrm{~g} / 100 \mathrm{~g})$, menos das formulações F4, F6 e F7 que os valores de proteínas obtidos na análise são a metade quando comparados com o fornecido pela tabela em questão; para lipídeos, verificou-se valores próximos aos analisados em relação a tabela $(3,25 \mathrm{~g} / 100 \mathrm{~g})$.

Com relação ao leite semidesnatado fornecido na Tabela de Composição de Alimentos da Escola Paulista de Medicina/Unifesp (2013), encontra-se valor energético de 50 $\mathrm{kcal} / 100 \mathrm{~g}$, o que se distancia das formulações, em média, 10 a $20 \mathrm{kcal}$.

Já para carboidratos, verificou-se na tabela valor de 4,8g/100g, e quando comparados aos resultados obtidos pelo presente estudo, as formulações F2, F4 e F5 apresentaram o dobro de carboidratos. Para valores de proteínas, o presente estudo reportou valores próximos aos apresentados pela tabela $(3,32 \mathrm{~g} / 100 \mathrm{~g})$, com exceção das formulações F4, F6 e F7 
onde os valores de proteínas foram abaixo do verificado na tabela. Rêgo et al. (2013)afirmam que muito embora sejam cumpridos os limites impostos para a densidade energética, a indústria debate-se com múltiplas dificuldades na modificação do leite de vaca, verificando-se dependência da proporção dos macronutrientes. Efetivamente a diferença entre valor energético suprido pelos macronutrientes é notória, percebendo-se que o leite humano apresenta3 vezes menos proteína, no entanto, quase o dobro de carboidratos, quando comparado ao leite de vaca. Innis (2003)relata que em comparação com o leite materno, as fórmulas lácteas apresentam maiores quantidades de proteínas e carboidratos, e menores de gordura, já o leite humano apresenta maior quantidade de gordura, sendo a maior fonte energética, formada por ácidos graxos essenciais como o ácido graxo araquidônico (AA) e o ácido docosahexaenóico (DHA), considerados benéficos para o desenvolvimento cerebral e acuidade visual.

\section{CONCLUSÃO}

Tendo em vista a consonância dos resultados obtidos neste trabalho, com os apresentados pela legislação vigente de fórmulas infantis, exceto quanto ao teor de lipideos que todas as formulações apresentaram valores inferiores em relação ao que a legislação preconiza, e mesmo constatando-se variações em relação as informações nutricionais fornecidas pelos fabricantes, evidencia-se que as as fórmulas infantis são substitutas ao leite materno e são nutricionalmente seguras, muito embora se associem a um crescimento, a um perfil bioquímico e de outros marcadores de saúde diferentes dos registrados em lactentes alimentados com leite humano.Os resultados obtidos apontam a necessidade de um contínuo monitoramento desses produtos por meio de programas de vigilância sanitária, uma vez que a inadequação dos nutrientes do teor lipídico e outros pode afetar de maneira significativa o desenvolvimento infantil.

\section{REFERÊNCIAS}

ANVISA. Agência Nacional de Vigilância Sanitária. Rotulagem Nutricional Obrigatória: Manual de Orientação às Indústrias de Alimentos. $2^{\mathrm{a}}$ versão. Brasília: Ministério da Saúde, 2005.

AOAC.Association of Official Analytical Chemists.Official Methods of Analysis of AOAC International.18. ed.Gaithersburg: AOAC, 2010.

BRASIL. Lei no 8069, de 13 de julho de 1990. Estatuto da criança e do 
adolescente. Brasília: Ministério da Saúde, 1991.

BRASIL. Ministério da Saúde. Agência de Vigilância Sanitária. Resolução RDC n ${ }^{\circ}$ 360, de 12 de dezembro de 2003. Aprova regulamento técnico sobre rotulagem nutricional de alimentos embalados, tornando obrigatória a rotulagem nutricional.Brasilia: 2003.

BRASIL. Ministério da Saúde. Agência de Vigilância Sanitária. Resolução RDC nº 44 de 19 de setembro de 2011. Dispõe sobre o regulamento técnico para fórmulas infantis de seguimento para lactentes e crianças de primeira infância, Brasilia; 2011.

BRASIL. Ministério da Saúde. Secretaria de Atenção à Saúde. Departamento de Atenção Básica. Saúde da criança: nutrição infantil: aleitamento materno e alimentação complementar. Brasília, 2009.

BRASIL. Ministério da Saúde. Secretaria de Política De Saúde. Organização Pan Americana De Saúde. Guia alimentar para crianças menores de dois anos. Brasília, 2002.

BRASIL. Ministério da Saúde. Secretaria de Vigilância Sanitária. Portaria ${ }^{\circ}$ 977, de 5 de dezembro de 1998. Regulamento técnico referente às fórmulas infantis para lactentes e às fórmulas infantis de seguimento. Diário Oficial da União. 1999.

EKPETE, O. A. et al. The nutrient value of breast milk and some infant formula. The Experiment, v. 8, n. 2, p.456-460, 2013.

ERHINYODAVWE, O.; EGELE, R. O.; IDOLOR, O.; UGBUNE, U. The nutrient value of breast milk and some infant formulae. Journal of Chemical Society of Nigeria, v. 34, n. 1, p. 64-67. 2009.

FITZPATRICK, J.; BARRY, K.; DELANEY, C.; KEOGH, K. Assessment of the flowability of spray-dried milk powders for chocolate manufacture.Le Lait, v. 85, n.4-5, p. 269-277, 2005.

IBGE. Instituto Brasileiro de Geografia e Estatística. Tabela de Composição Nutricional dos Alimentos Consumidos no Brasil; Rio de Janeiro 2011.

INNIS, S.M. Perinatal biochemistry and physiology of long-chain polyunsaturated fatty

acids. The Journal of Pediatrics, v. 143, n. 4, p. 1-8, 2003.

INSTITUTO ADOLFO LUTZ. Normas Analíticas do Instituto Adolfo Lutz: Métodos químicos e físicos para análises de alimentos.IV Ed. São Paulo: IMESP, 2005.

MERRIL, A.L; WATT, B.K. Energy value of foods: basis and derivation.Washington: United States Department of Agriculture, 1973.

\section{PALMER, G. The Politics of} Breastfeeding: When Breasts are Bad for Business. London: Pinter and Martin. 2009.

RÊGO, C.; TELES, A.; GUERRA, A.; NAZARETH, M. Leites e fórmulas infantis: a realidade portuguesa revisitada em 2012. ActaPediátrica Portuguesa, v. 44, n. 5, p. 50-93, 2013.

SEMENIUC, A.; SEVASTIŢA, M; ANCUTA, R.M.; SUHAROSCHI, M.; TOFANA, M. Influence of the storage conditions on physicochemical parameters of infant formula.JournalofAgroalimentary 
Processes and Technologies, v. 18, n.1, p.

61-64.

TAVARES, C.S.C. Estabilidade físicoquímica de fórmulas infantis, 2013

[acesso em 13 set 2014]. Disponível em: https://iconline.ipleiria.pt/bitstream/10400. 8/1077/1/Projeto\%20Cl\%C3\%Aludia\%20 Tavares.pdf;

UNIFESP. Tabela de Composição de Alimentos da Escola Paulista de

Medicina, 2013 [acesso em 10 out 2014].

Disponível em:

http://www2.unifesp.br/dis/servicos/nutri/p ublic/ 